\title{
EXPERIMENTAL PERFORMANCE EVALUATION AND FEASIBILITY STUDY OF 6LOWPAN BASED INTERNET OF THINGS
}

\author{
James AGAJO*, Jonathan G. KOLO*, Mutiu ADEGBOYE**, Bello NUHU*, Lukman AJAO*, Ibrahim ALIYU* \\ *Department of Computer Engineering, Federal University of Technology, Minna Niger State, Nigeria \\ ${ }^{* *}$ Department of Computer Engineering Federal University Oye Ekiti, Nigeria
}

\begin{abstract}
Nowadays, demand for low power, small, mobile and flexible computing machines that interconnects are growing rapidly. This study highlights internet of things (IoT) model regarding sensor node discovery and IPV6 framework using 6LoWPAN. Contiki network simulator (cooja) was used to examine the performance of the proposed network. The simulator was chosen because it provides good graphical user interface environment and allow rapid simulation setup found to be best in simulating network involving 6LoWPAN. Three experiments were carry out with the network topology designed to have 3, 7 and 5 motes respectively. The parameters considered in the simulation were throughput and packet loss which were examined using packet generation rate of 1 to 50 packet/sec with a constant delay. GET requests was sent to the humidity and temperature sensor motes running CoAP servers, and the corresponding throughput were observed in each case per experiment, it was observed that there was a 10 packet per second increase before it finally dropped This was because of the packet loss due to the increase in traffic. GET request was sent to motes to obtain the packet loss and the packet that were not acknowledged determined the packet loss. In this study, the performance of the proposed model in terms of throughput and packet loss was studied and the expected results will aid in planning 6 LoWPAN network, A transition flow diagram was evolved for this work to represent packet routing process.
\end{abstract}

Keywords: internet of things, network, throughput, packet loss, IPv6, 6LoWPAN

\section{INTRODUCTION}

Internet of things (IoTs) can be refers to as a model for connecting objects, peoples and things to the internet to enhance their ability to communicate with one another [1]. IoTs are made up of network of physical objects with entrenched technology to sense and communicate their external or the internal environments. The idea of IoTs spans through many applications, systems and protocols otherwise known as "things" [2]. Environmental sensors enable the monitoring of environmental condition either to know the present environmental state or providing early warnings.

As some of these sensors are part of the objects in the network, it is required to connect them to the internet using standard internet protocol across the sensor boundary to increase the access to the data they acquire. Things that are manually operated can now be accessed and visualized automatically [3].

To enable sensor network function for a reasonable period of time, the power consumption is an important factor. Most of the hardware and software design has effect on sensor node power usage [4]. Communication is one of the major energy consuming factor. Adoption of efficient wireless protocol transmission and reception period is important. As a result, power consumption can be reduced. This will enable CPU and radio to be more stable at off/standby state. Consumption efficiencies in the radio protocol make a noteworthy difference to the power consumption of the node. Initial data processing on the nodes can moderate the amount of data required to be sent, but CPU required longer operation cycle to process the reading task [5].

Though, not all the sensor network or nodes in a network required power control. However in some cases, Sensor nodes powered with main power supply could still be backed up with recharge batteries, a typical example is the smart grid sensors in power systems [6]. Some of the present sensor network employed one or more gateway for interfacing with the network, handle collection of data and uploading to the external location [7]. The challenge of such system is that there is need for the gateway to know about everything within the network.

Communication protocol standard has been fashioned around the protocol and technology of internets. As sensor networks connected to this infrastructure they can communicate far beyond the perimeter of their own network. Under the banner of internet of things technologies for internet connected sensor systems has been proposed [8].

Recently, some devices are implementing sensor discovery mechanism and Internet Protocol version 6 (IPv6) frameworks. However, most of these mechanisms have been considered for local area networks (LANs) and likewise expanded for IPv6 over Low Power Wireless Personal Area Network (6LoWPAN). Some of these mechanisms including Universal Plug and Play (UPnP) protocol which permits automatic creation of device-todevice connection, and Service Location Protocol (SLP) that can enable devices to communicate in LAN without previous configuration. SLP can be use by the Devices to announce their availability in the local network [9]. This is important for large-scale internet of things scenario.

In computer networks, devices are discovered based on the protocols they are supported on and the rules in the network. For example, Address Resolution Protocol (ARP) has been used for hosts discovering on a local area network [10]. At the device and service levels, mechanisms such as Semantic Web Services [11] and Universal Plug and Play (UPnP) [12] have been supported. Device discovery has been inspected and applied in both local and mobile network intensively [12], [13], [14]. However, in IoTs scenario, technology which could enhance the discoverability of device with low power consumption is required to be adopted. 


\section{IOTS COMMUNICATION}

Various potential technologies have been in existence for bringing the internet to IoTs. Some of these technologies are from existing internet connectivity options, whereas others are modified from sensor network protocols to new IP based connection systems [15]. However, the challenges of this developments are the issue of addresses. Research by [16] indicate that up to 26 billion of unit will be interconnect by 2020 through the IoTs. Using IPv4 address show that only 4,294,967,296 address will be available. To evade problem of shortage of address, adoption of protocol extension that will make connection of large number of device possible is required.

IPv6 is dedicate to provides 2128 addresses, using this protocol will make prediction of 26 billion of unit that will be connected in 2020 become reality [16]. Asides from large numbers of IPv6 addresses, there are features of IPV6 that are applicable and can be deployed in IoT models. However, shortcoming such as issue of higher computation and packet compression require addressing. Nevertheless, an IP-based technology for the IoTs network is preferred because of the following features:

1) Compatibility,

2) Connectivity,

3) Mature technology, and

4) Open.

\section{A. 6LoWPAN}

6LoWPAN network [17] is a low-cost communication networks that enable IPv6 connectivity. 6LoWPAN fashioned devices are compatible with IEEE 802.15.4 standard [18], it features are low cost, short range, low memory usage and low bit rate [19]. IPv6 is not fully supported in Wireless sensor network (WSN) [19] due to limited available frame size on WSN [20]. The available frame size on WSN is 127 bytes, and IPv6 has 40 bytes of a fixed header size. This indicate overhead levied by many headers would reduce size of byte left for transmission of data in WSN.

6LoWPAN comprise of edge router and sensor nodes (6LoWPAN). Edge router is the core of 6LoWPAN network that link 6LoWPAN network to the other IP internet. It is responsible for routing 6LoWPAN packet to the IPv6 packet, and assigning IPv6 prefixes in the 6LoWPAN network. The routing mechanism for 6LoWPAN network are: mesh-under and route over [21].

The mesh-under router mechanism permits layer 2 routing, whereas route-over mechanism routing with the aids of network layer [22].

The maximum transmission packet size in a standard IPv6 is 1024 bytes [23]. Whereas it is reduced to 127 bytes in 6LoWPAN. This illustrate why IPv6 packet does not fit into an IEEE 802.15.4 frame. In IPv6 network, 40 bytes is reserved for IPv6 header, 25 bytes for MAC header, additional 8 bytes may also be used for UDP headers. This will make left-over payload to be about 54 bytes in 6LoWPAN. To offset for small available frame size of a payload, adaptation layer has been established by IETF to reduce IPv6 overhead header [17].

High IPv6 header compression, Bootstrap, multihoming and flexibility are some of the important feature of 6LoWPAN. The existences of edge routers in the 6LoWPAN which share the same IPv6 prefix is the core difference between simple and extended 6LoWPAN [24].

\section{B. Network Discovery of embedded Devices}

The central process in distributed and ubiquitous computing is network discovery. Network discovery enable automatic advertising and registering of the services on the network.

With the intent to enhance 6LoWPAN feature, various protocols have been proposed among them are: Universal Plug and Play (UPnP), Service Location Protocol (SLP), Device Profile for Web Services, and Sun's Jini, or Apple's Bonjour. Some of these protocol have been incorporated in some new developed devices to enable auto-setup of service discovery for the end users.

Service discovery is the mechanism that is important in an event where devices are required to join the network and service running [25]. Various process for device to finding services that are running are as follows:

RESTful active network discovery, where devices advise its presence to the LDU automatically.

WS-Discovery on which DPWS is based both active and passive.

Passive RESTful discovery for REST-enabled devices that do not comply with SIA network discovery.

\section{PROPOSED NETWORK FOR 6LOWPAN BASED IOTS MODEL}

The proposed network architecture for 6LoWPAN based sensor node discovery for IoTs paradigm is depicted in Fig. 1. The architecture made up of 6LoWPAN nodes and edge router that functions without gateway software. These means data can be exchange freely by using edge router as a simple gateway.

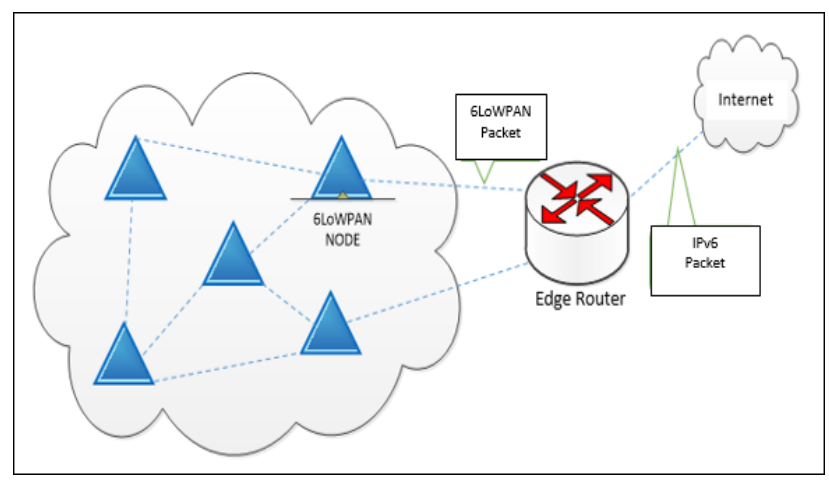

Fig. 1 Proposed Network Architecture

Fig. 1 shows an example of an IPv6 network, including a 6LoWPAN mesh network. The uplink to the Internet is handled by the Access Point (AP) acting as an IPv6 router. Several different devices are connected to the AP in a typical setup, such as PCs, servers, etc.

We adopt some form of Algorithmic State Machine (ASM) to represent the packet Routing process layout, which is in the form of a flowchart, a logical condition is written in each conditional vertex. It is possible to write the same logical condition in different conditional vertices shown in Fig. 3. 


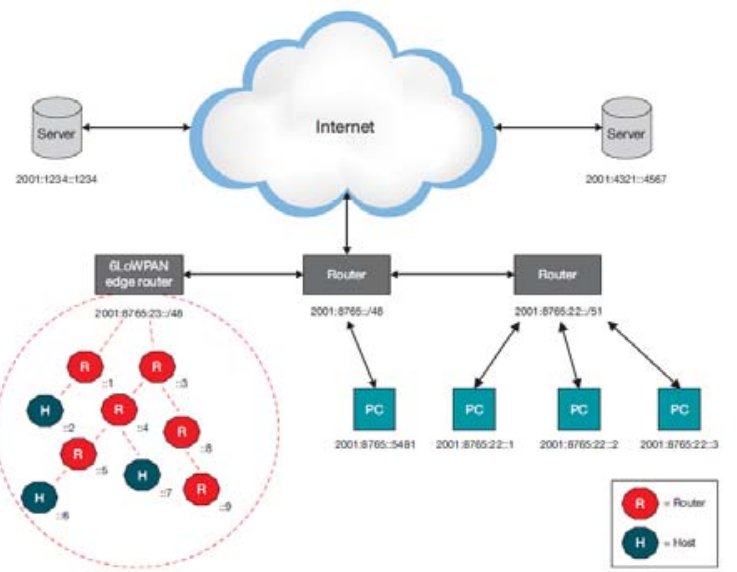

Fig. 2 An example of an IPv6 network with a 6LoWPAN mesh network [26]

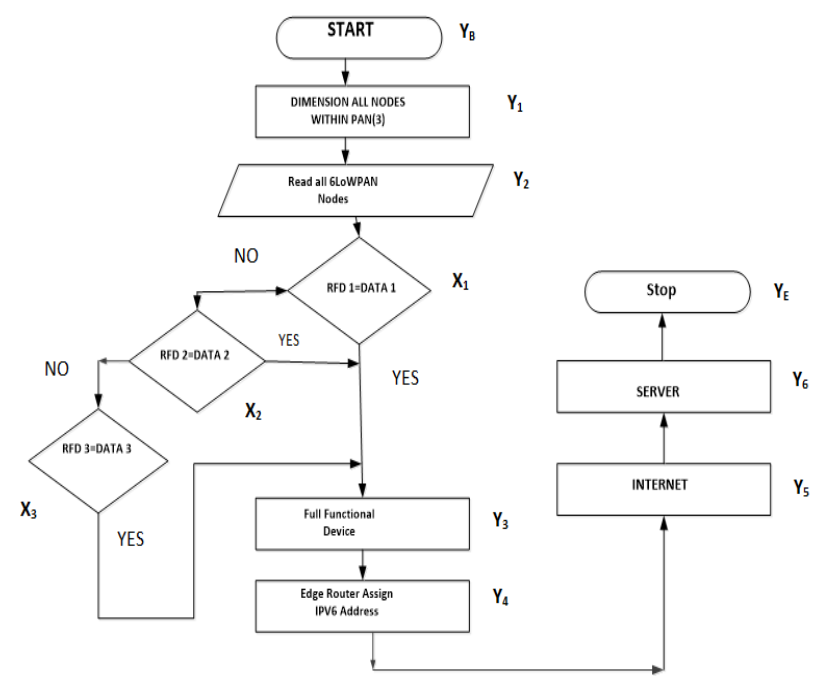

Fig. 3 Flow Diagram of Packet routing Process

A microinstruction (an operator), containing one, two, three or more operations, is written in each operator vertex of the flowchart. It is possible to write the same operator in different operator vertices.

If we replace logical conditions by $\mathrm{X} 1, \mathrm{X} 2, \ldots, \mathrm{X} 3$, and operators by $\mathrm{Y} 1, \mathrm{Y} 2, \ldots, \mathrm{Y} 6$.

An equation was evolved to represent a flow diagram which we refer to as transition flow equation 1 and 2 respectively.

$\left(Y_{B} Y_{E}\right)=Y_{1}+Y_{2}+X_{1}+Y_{3}+Y_{4}+Y_{5}+Y_{6}$

$\left(Y_{B} Y_{E}\right)=Y_{1}+Y_{2}+X_{1}+X_{2}+X_{3}+Y_{3}+Y_{4}+Y_{5}+Y_{6}(2)$

Equation 1 and 2 are different route for packet transmission from the Beginning YB to the End YE. X' represent logical vertex decision NO, $\mathrm{X}$ represent logical vertex decision YES.

A Signal-to-Interference Ratio Analysis for the Node Links, which will model the node link, small-scale (such as Rayleigh Fading) model and large-scale power low propagation model were considered. By considering the transmitter-to-receiver distance dtr and transmitting power $\mathrm{Pt}$, the received signal power $\mathrm{Sp}$ can be obtain using mathematical model in equation 3.
$S \_p=P \_t . h \_i j . \llbracket d \_t r \rrbracket \wedge(-\propto)$

Where hij represent exponentially distributed fading coefficient for standard path loss coefficient and subject to constraint $\alpha=2$.

To determine the Successful Transmission Probability (STP) of the node to node links, mathematical model in 3.2 was employed as the probability that the quality of a packet received $\mathrm{Pr}$ denote probability that packet successfully reach expected threshold $\mu$.

$\mathrm{P} \_\mathrm{r}=\left(\mathrm{P} \_\mathrm{s}-\mathrm{P} \_\mathrm{d}\right)>\mu$

Where Ps represent packet send and Pd represent packet drop.

\subsection{Experimental Setup}

The simulation setup was design to examine RPL routing and to evaluate the performance of the stack. Three experiments were setup as presented in Fig. 4a), $4 b), 4 c)$. The design consists of 3,5 and 7 motes respectively, running CoAP servers providing humidity and temperature values as resources from the fundamental SHT11 sensors.

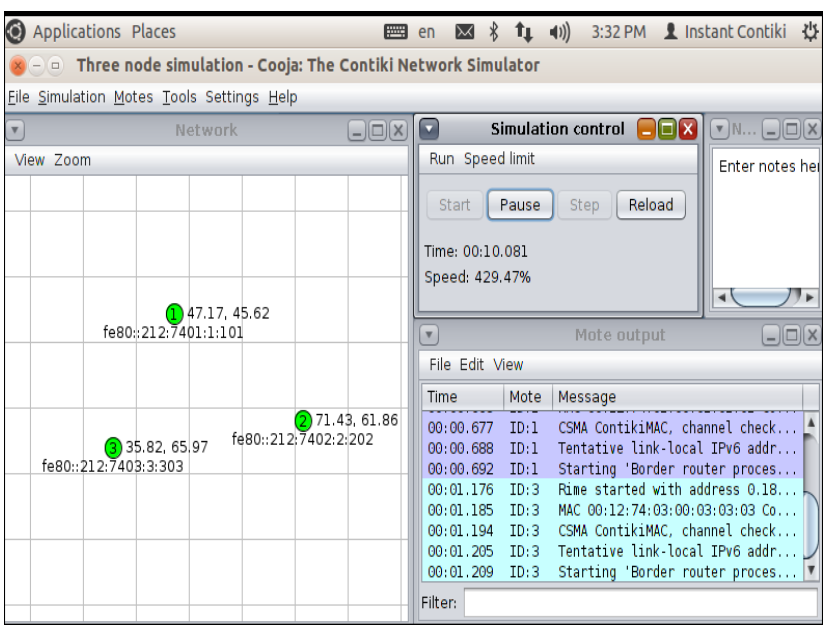

Fig. 4a) Simulation Scenario 1

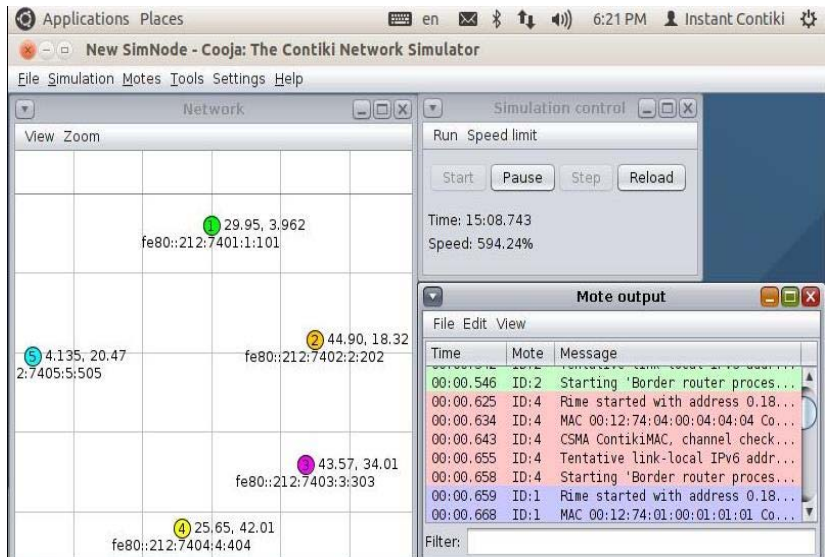

Fig. 4b) Simulation Scenario 2 


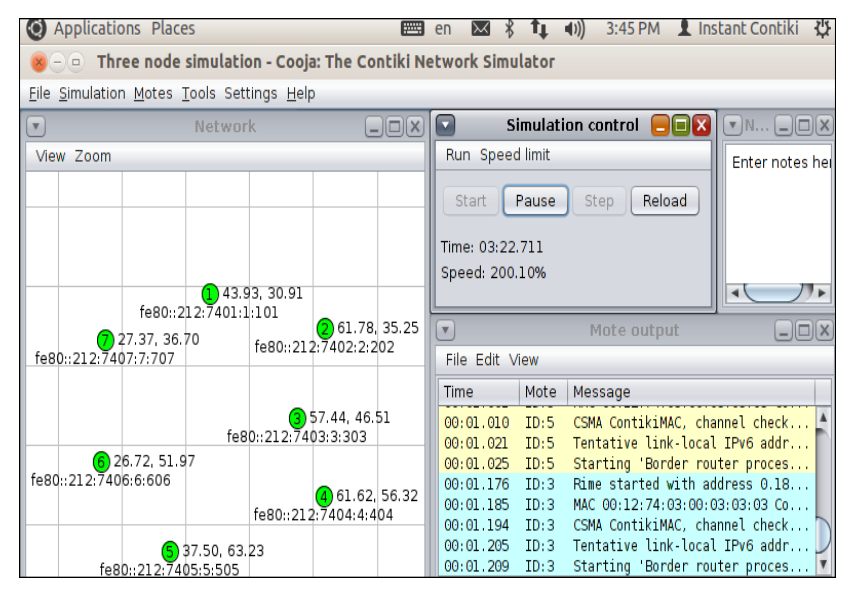

Fig. 4c) Simulation Scenario 3

The selected motes were queried periodically through the CoAP client. The GET requests were sends to the servers in the simulation setup to acquire humidity as well as temperature values.

In each of the setup, multiple motes running CoAP servers were selected. Table 1 present Selected simulation parameters with their corresponding values.

Table 1 Simulation parameters

\begin{tabular}{|l|l|}
\hline Name of Selection Parameter & \multicolumn{1}{|c|}{ Values } \\
\hline Radio medium & $\begin{array}{l}\text { Unit Disk Graph } \\
\text { Medium }\end{array}$ \\
\hline Mote Startup delay & $1000 \mathrm{~ms}$ \\
\hline Random seed & 61728 \\
\hline AC layer & CSMA/CA \\
\hline Mote type & T mote Sky \\
\hline Bit rate & $240 \mathrm{kbps}$ \\
\hline Radio duty cycling & NullRDC \\
\hline Node duty cycle & $100 \mathrm{~m}$ \\
\hline Node carrier sensing range & $100 \mathrm{~m}$ \\
\hline Max transmit wait & 6975 \\
\hline Ack timeout & 1200 \\
\hline Ack random factor & 1.2 \\
\hline Tx/Rx ration & $100 \%$ \\
\hline
\end{tabular}

\section{ANALYSIS AND DISCUSSION}

During the simulation process, the parameters considered were throughput and packet loss for the selected nodes in the network. The bound of the RPL routing protocol using 6LoWPAN as the setup feature mote with the different nodes to the router was evaluated in each of the setup. The throughput values obtained for each of the experiment were presented in the Fig. 4, 5 and 6 respectively.

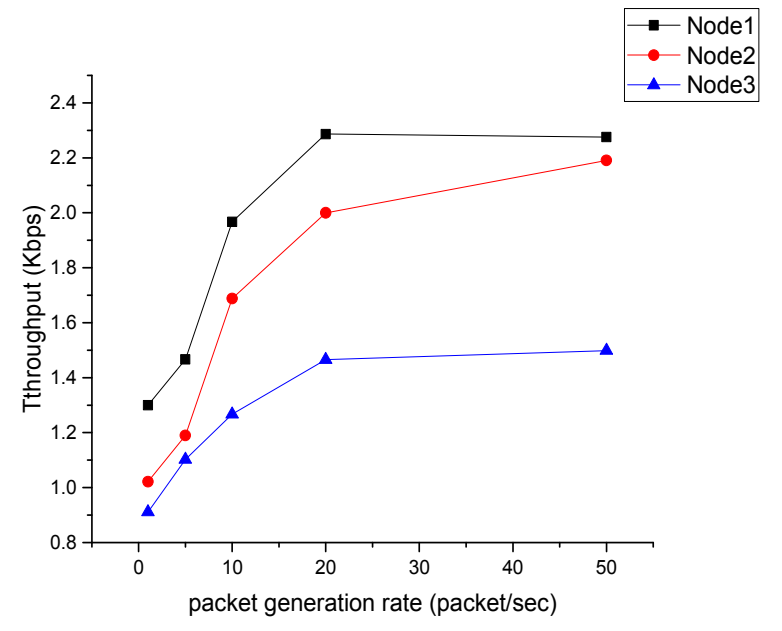

Fig. 4 Throughput vs Packet Generation Rate (Exp. I)

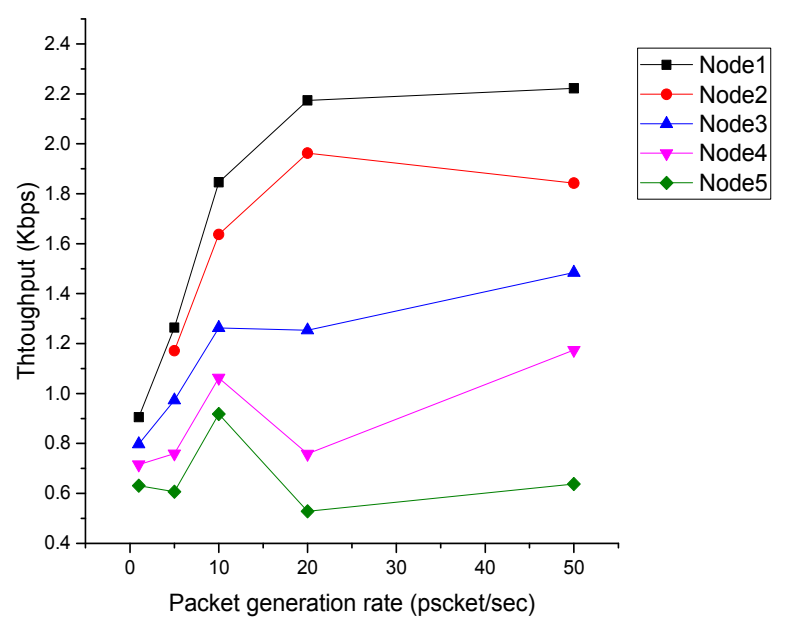

Fig. 5 Throughput vs Packet Generation Rate (Exp. II)

Table 2 Packet Loss against Packet generation rate

\begin{tabular}{|c|c|c|c|c|c|c|c|c|}
\hline SN & $\begin{array}{c}\text { PACKET } \\
\text { LOSS }\end{array}$ & $\begin{array}{c}\text { PACKET } \\
\text { LOSS }\end{array}$ & $\begin{array}{c}\text { PACKET } \\
\text { LOSS }\end{array}$ & $\begin{array}{c}\text { PACKET } \\
\text { LOSS }\end{array}$ & $\begin{array}{c}\text { PACKET } \\
\text { LOSS }\end{array}$ & $\begin{array}{c}\text { PACKET } \\
\text { LOSS }\end{array}$ & $\begin{array}{c}\text { PACKET } \\
\text { LOSS }\end{array}$ & $\begin{array}{c}\text { PACKET } \\
\text { LOSS }\end{array}$ \\
\cline { 2 - 9 } & NODE 1 & NODE 1 & NODE 2 & NODE 3 & NODE 4 & NODE 5 & NODE 6 & NODE 7 \\
\hline 1 & 1.0 & 1.0 & 1.0 & 4.0 & 5.1 & 7.0 & 7.1 & 7.0 \\
2 & 5.0 & 2.0 & 6.0 & 7.0 & 12 & 13 & 16 & 16 \\
3 & 10 & 3.5 & 7.5 & 1.2 & 15 & 21 & 26 & 26 \\
4 & 15 & 4.0 & 7.4 & 13 & 15 & 22 & 26 & 28 \\
5 & 20 & 5.0 & 6.0 & 14 & 17 & 21 & 27 & 29 \\
6 & 25 & 6.5 & 8.0 & 12 & 18 & 22 & 26 & 31 \\
7 & 30 & 7.0 & 8.1 & 16 & 20 & 23 & 30 & 32 \\
8 & 35 & 7.1 & 10 & 17 & 22 & 25 & 33 & 32.5 \\
9 & 40 & 7.3 & 11 & 17.5 & 25 & 22 & 35 & 33 \\
10 & 45 & 7.5 & 12 & 18 & 23 & 27 & 33 & 34 \\
11 & 50 & 8.0 & 13.5 & 20 & 26 & 30 & 34 & 35 \\
\end{tabular}


From the obtained result, it is observed that throughput raised at about 10 packets per second and then declines. This can be characterized to occurrences of greater packet loss due to increase in incoming traffic.

Packet loss frequently occurs as a result of atmospheric noise. This experiment was carried out and validated in a cooja environment by varying the $\mathrm{Tx} / \mathrm{Rx}$ ratio (in \%). The packet loss that are not acknowledged determined the packet Table 2 is a breakdown of the packet generated and packet loss for the nodes.

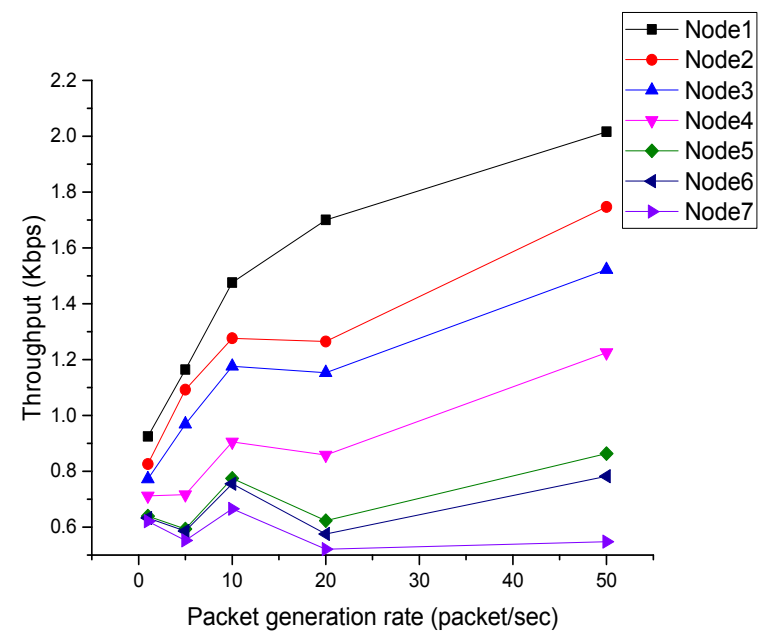

Fig. 6 Throughput vs Packet Generation Rate (Exp. III)

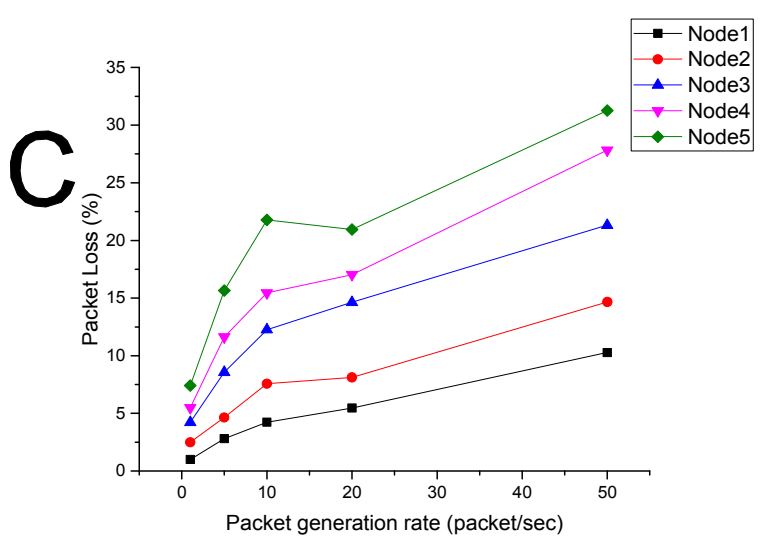

Fig. 7 Packet loss vs Packet Generation Rate (Exp. IV)

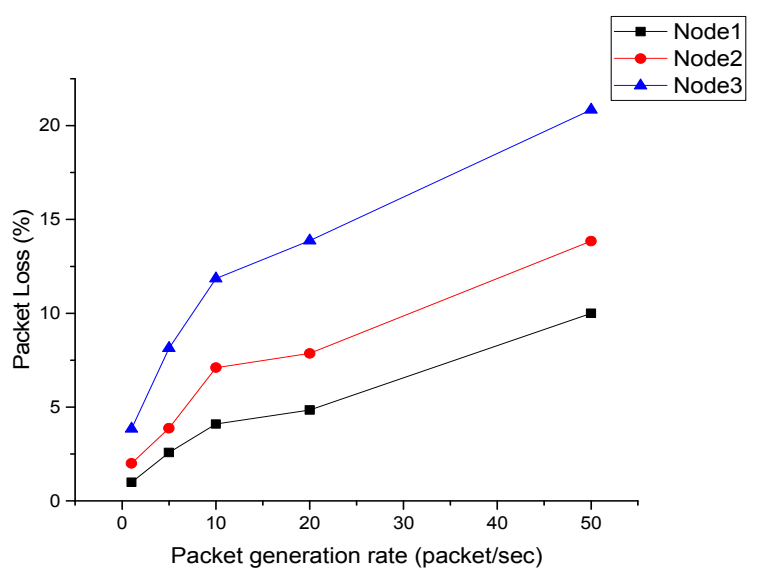

Fig. 8 Packet Loss vs Packet Generation Rate (Exp. V)
Fig. 4, 5, 6, which represents packet throughput against packet generated while 7,8 and 9 represent packet loss against packet generated respectively illustrate graphical representation of the obtained result. The obtained result indicate packet loss increase with increase in nodes, this is due to distance between each node and some other factor that link to an environment where the network is established.

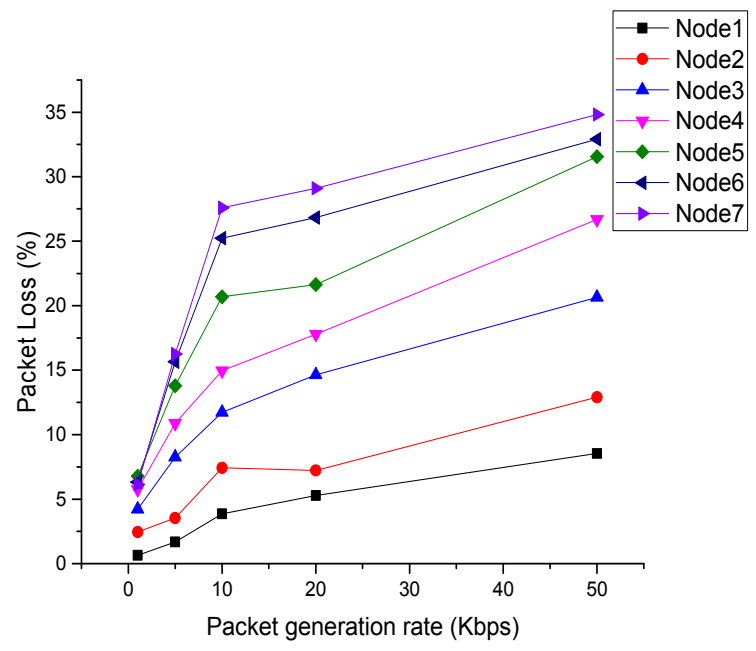

Fig. 9 Packet loss versus packet generation rate (Exp. VI)

\section{CONCLUSIONS}

This research work carried out analysis and evaluation of 6LoWPAN based Internet of Things with a view to coming up with the feasibility of realising the application as it relates to environmental monitoring. This study highlights internet of things (IOT) model regarding sensor node discovery and IPV6 framework using 6LoWPAN. Contiki network simulator (cooja) was used to examine the performance of the proposed network. The simulator was chosen because it provides good graphical user interface environment and allow rapid simulation setup found to be best in simulating network.

The result obtained for both temperature and humidity in terms of throughput and packet loss were useful for predicting the performance and characterising of the proposed networked.

It is in our best interest that the research work will be helpful in future researched as it relates to Wireless Sensor Network and Internet of Things.

\section{ACKNOWLEDGMENTS}

We wish to acknowledge and appreciate the authorities of the Federal University of Technology for fortifying our research with the recently procured wireless Sensor nodes and establishment of Wireless Sensor Test bed, where different experiment are now been carried out and deployed in the department of Computer Engineering.

\section{REFERENCES}

[1] ATZORI, L. - IERA, A. - MORABITO, G.: The Internet of Things: A survey, Computer Networks, 54 (2010), pp. 2787-2805. 
[2] LEHONG, H. et al.: Hype Cycle for Emerging Technologies, Gartner, 2014.

[3] KRANZ, M. - HOLLEIS, P. - SCHMIDT, A.: Embedded interaction: Interacting with the internet of things, IEEE Internet Computing, 14 (2010), pp. 46-53.

[4] KO, J. et al:: Low power or high performance? A tradeo whose time has come (and nearly gone), in Wireless Sensor, 2012. pp. 98-114.

[5] KIMURA, N. - LATI, S.: A survey on data compression in wireless sensor networks, in International Conference on Information Technology: Coding and Computing, 2005, pp. 8-13.

[6] FARHANGI, H.: The path of the smart grid, in Power and Energy Magazine, IEEE, 2010, pp. 18-28.

[7] MARTINEZ, K. et al.: Field deployment of low power high performance nodes, in The Third International Workshop on Sensor Networks (SN 2010), 2010.

[8] DURVY, M. et al.: Making sensor networks ipv6 ready. in Proceedings of the 6th ACM conference on Embedded network sensor systems, SenSys '08, New York: ACM, 2008.

[9] CIRANI, S. et al.: A Scalable and self-Configuring Architecture for Service Discovery in the Internet of Things, IEEE Internet of Things Journal, 1(5) 2014, pp. 508-521.

[10] PLUMMER, D. C.: RFC 826: An ethernet address resolution protocol, InterNet Network Working Group, 1982.

[11] KLUSCH, M. - FRIES, B. - SYCARA, K.: Automated semantic web service discovery with OWLS-MX, in Proceedings of the Proceedings of the fifth international joint conference on Autonomous agents and multiagent systems, 2006, ACM.

[12] JERONIMO, M. - WEAST, J.: UPnP design by example, Intel Press, 2003.

[13] AHAMED, S. I. - ZULKERNINE, M. ANAMANAMURI, S.: A dependable device discovery approach for pervasive computing middleware. Proceedings of the First International Conference on Availability, Reliability and Security (ARES), 2006.

[14] OUTAY, F. - VÈQUE, V. - BOUALlÈGUE, R.: Survey of Service Discovery Protocols and Benefits of Combining Service and Route Discovery, IJCSNS, 7 (11) 2007.

[15] GUBBI, J. et al.: Internet of Things (IoT): A vision, architectural elements, and future directions, Future Generation Computer Systems, 29 (7) 2013, pp. 1645-1660.

[16] LEO, M. et al.: A Federated Architecture Approach for Internet of Things Security, in Euro Med Telco Conference (EMTC), 2014, pp. 1-5.
[17] HUI, J. - THUBERT, P.: Compression Format for IPv6 Datagrams over IEEE 802.15.4-Based Networks, 2011.

[18] IEEE Standard (802.15.4), Wireless medium access control and physical layer specications for low-rate wireless personal area network, 2009.

[19] ROTH, D. - MONTAVONT, J. - NOEL, T.: Performance evaluation of mobile IPv6 over 6LoWPAN, in Proceedings of the 9th ACM symposium on Performance evaluation of wireless ad hoc, sensor and ubiquitous networks, PEWASUN '12, 2012, New York, USA.

[20] SHENG, Z. et al.: A survey on the ietf protocol suite for the internet of things: Standards, challenges, and opportunities, IEEE Wireless Communications, 20 (6) 2013, pp. 91-98.

[21] DJAMAA, B. et al.: Towards efficient distributed service discovery in low-power and lossy networks. Wireless Networks, 20 (8) 2014, pp. 2437-2453.

[22] SHELBY, Z. - BORMANN, C.: The Wireless Embedded Internet, 2009.

[23] SILVA, R. - SILVA, J. S. - BOAVIDA, F.: Evaluating 6LoWPAN implementations in WSNs, Proceedings of 9th Conferncia sobre Redes de Computadores Oeiras, Portugal, 2009.

[24] KUMAR, V. - TIWARI, S.: Routing in IPv6 over low-power wireless personal area networks (6LoWPAN): A survey, Journal of Computer Networks and Communications, 2012.

[25] GUINARD, D. et al.: Interacting with the soa-based internet of things: Discovery, query, selection, and on-demand provisioning of web services, IEEE transactions on Services Computing 3, 2010, pp. 223-235.

[26] OLSSON, J.: 6LoWPAN demystified, System Application Engineer, Texas Instrument, 2014.

Received February 24, 2017, accepted May 2, 2017

\section{BIOGRAPHIES}

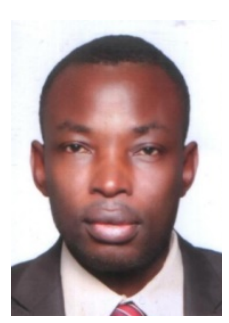

Dr. James Agajo is a senior lecturer and also a highly motivated career driven achiever with over Seventeen (17) years working experience in the field of Telecommunication, Spec-trum Mana-gement, Computer, Mechatronics Engineering, Industrial Automation, Teaching, Research and Project Development and Signal Processing. However his main areas of research includes Wireless Sensor Network, Wireless Smart System, Computer network, Intelligent System design, Digital Signal Processing (DSP), Artificial intelligence Support Vector Machine, Supervised learning, Internet of Things (IOT) and Embedded systems. Dr James Agajo received a Bachelor of Engineering (B.Eng.) Degree in Electrical and Computer Engineering from the 
Federal University of Technology, Masters of Engineering (M.Eng.) Degree in Electronic and Computer Engineering from the Nnamdi Azikiwe University, (Ph.D) in Telecommunication and Computer Engineering from Nnamdi Azikiwe University Awka Nigeria. Dr. James Agajo is a Lecturer with the Department of Computer Engineering Federal University of Technology Minna, Niger State Nigeria. He has many publication to his record at national and international level, he is a member of many Bodies amongst which are IASCIT, MIRDA, MNSE, COREN Reg., IAENG.

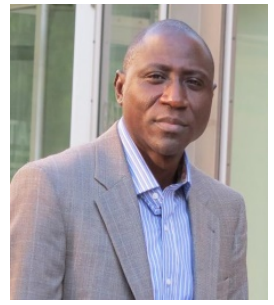

Engr. Dr. Jonathan Gana Kolo received Bachelor of Engineering (B.Eng.) Degree in Electrical Engineering from Ahmadu Bello University, Zaria; Masters of Science (M.Sc.) Degree in Electrical and Electronic Engineering (Telecommunication option) from the University of Lagos, Lagos; Doctor of Philosophy (Ph.D) Degree from The University of Nottingham Malaysia Campus. He is a Registered Engineer with the Council for the Regulations of Engineering in Nigeria (COREN), and a Corporate Member of the Nigerian Society of Engineers (NSE). His research interests are majorly in the areas of Wireless Sensor Networks, Embedded Systems, Electronics, Digital Signal Processing, Intelligent Systems, Digital Image processing and Communication Engineering. His Research results are published in several peer-reviewed papers. Dr. Kolo is a recipient of MISTI (MIT International Science and Technology Initiatives) Africa Empowering the Teachers (ETT) Fellowship of the Massachusetts Institute of Technology, USA.

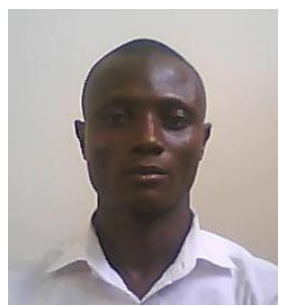

Nuhu Bello Kontagora is currently an Assistant Lecturer (AL) in the department of Computer Engineering, Federal University of Technology Minna, Nigeria. He obtained his first degree (B.Eng.) in Electrical /Computer Engineering from the same institution in the year 2010. He obtained his second degree (M.Tech) in
Computer Science and Engineering at Ladoke Akintola University of Technology Ogbomoso, Nigeria. His research interests include: Artificial and Computational Intelligence, localization in sensor networks, wireless network security and Software Defined Networking. Some of his research are published in reputable journals and conference proceedings.

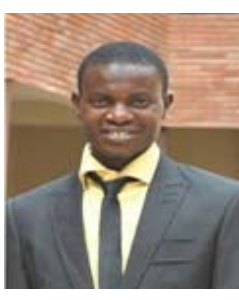

Mutiu Adesina Adegboye received his B.Eng. degree in Computer Engineering, Federal University of Technology Minna, Nigeria. $\mathrm{He}$ is currently doing his Master degree in the same department. His area of interest are Signal Processing, Machine Learning, Embedded System and

Internet of Things.

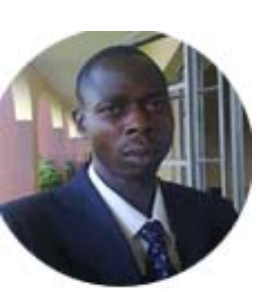

Lukman Adewale Ajao is a Senior Technological Researcher. $\mathrm{He}$ is currently working in the Department of Computer Engineering, Federal University of Technology, Minna, Nigeria. He obtained Postgraduate Diploma in Computer Science (PGDCS) in 2013 from University of Ilorin, Ilorin, Nigeria. He is currently a Master of Engineering research student in the Department of Computer Engineering, Federal University of Technology, Minna. The research interest are Embedded System based Internet of Things, Wireless Sensor Network, FPGA and Networking.

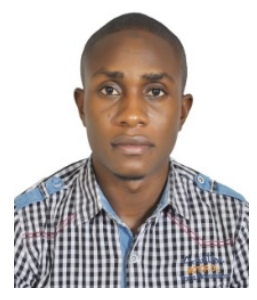

Ibrahim Aliyu received his B.Eng. degree in Computer Engineering from Federal University of Technology, Minna in 2014. He is currently working towards M.Eng. degree in Computer Engineering in computer Engineering in the same institution. His research interest includes Software Defined Network (SDN), Computer Network Security, Computitional Intelligence and Wireless Sensor Networks. 